Ann. Biol. anim. Bioch. Biophys., 1978, 18 (1), 201-210.

\title{
Ostéochondrose et faiblesse des pattes chez le porc
}

\author{
par A. POINTILLART, L. GUEGUEN
}

Station de Recherches de Nutrition, I.N.R.A.

78350 Jouy en Josas.

Summary. Osteochondrosis and leg weakness in swine.

Osteochondrosis seems to play a major role in the complex etiology of the leg weakness syndrome in swine. The present review discusses recent work on the subject. The main etio-pathogenic factors are shown to be of a mechanical nature, favorized by a morphological and genetic predisposition and aggravated by bad rearing conditions. Nutritional factors, especially dietary minerals, seems to have a secondary role.

The various anatomopathological aspects of the syndrome are described and emphasis is put on the frequency of lesions, which may reach 100 p. 100 in selected pigs at slaughter and on their preferential localization at some epiphyseal plates and joints. The presence of characteristic lesions is not always expressed by leg weakness disorders.

La fréquence des troubles de la locomotion chez certains types génétiques de porcs dans des conditions intensives d'élevage s'est considérablement accrue depuis une dizaine d'années. Les conséquences économiques de ce « syndrome de la faiblesse des pattes 》 (SFP) ou « leg weakness 》 des anglo-saxons, sont très importantes et aucune solution préventive ou thérapeutique n'existe actuellement.

Le but de ce bref rapport n'est pas de dresser un inventaire exhaustif de l'incidence, des causes possibles et des conséquences de ces anomalies, qui ont déjà fait l'objet de plusieurs excellentes revues de synthèse, dont l'une en France par Lefebvre et al. (1975) à laquelle nous ferons de larges emprunts. Parmi les principales publications portant sur ce problème il convient de citer en particulier les travaux britanniques (Walker ef Jones, 1962 ; Walker ef al., 1966 ; Duthie et Lancaster, 1964 ; Vaughan, 1969 et 1971 ; Fell ef al., 1970), allemands (Sabec ef al., 1961 ; Grünhagen ef al., 1970 ; Stadie, 1971 ; Teuscher, 1972 ; Koch et Meister, 1972 ; Herrmann, 1972 ; Ghaffari-Kaschani, 1973 ; Röpke, 1973 ; Pfleiderer, 1973 ; Afschar-Hamdi, 1973), norvégiens (Grøndalen, 1974a, b, c, d, e, f, g, h et 1976 ; Grøndalen et Vangen, 1974) et hollandais (Goedegebuure, 1976).

Le spectre étiologique de ce syndrome est très large mais seuls seront évoqués les troubles aseptiques ostéo-articulaires, à l'exclusion des troubles locomoteurs ayant leur origine ou leur effet en dehors de l'os : système nerveux, muscles, tendons, ligaments, pieds ef onglons, ou provenant de blessures ou d'infection.

Selon Grøndalen (1976), les lésions d'ostéochondrose, d'arthrose, d'épiphysiolyse ou de dégénérescence des disques intervertébraux seraient responsables de 
75 p. 100 des cas sévères de faiblesse des pattes observés en Norvège sur les porcs reproducteurs. Les troubles articulaires chez le Verrat se situent surtout aux niveaux du coude ef du grasset ( 30 p. 100$)$, des vertèbres lombaires $(30$ p. 100$)$ et de la tête du fémur (15 p. 100). Les lésions ostéo-articulaires sont donc en grande partie responsables de la faiblesse des pattes et il est logique de considérer surtout l'ostéochondrose, trouble de l'ossification endochondrale au niveau du cartilage de conjugaison ou du cartilage articulaire, pouvant évoluer en épiphysiolyse ou en arthrose.

Il importe cependant de constater, avec Grøndalen (1976), que l'incidence de l'ostéochondrose est presque de 100 p. $100 \mathrm{chez}$ les porcs « modernes » ef qu'elle est encore très élevée chez les porcs à faible vitesse de croissance, ce qui ne signifie pas qu'il y ait toujours faiblesse des pattes. Seule l'ostéochondrose sévère, avec des lésions « ouvertes», peut donner lieu d̀ des signes cliniques.

\section{Facteurs étio-pathogéniques de l'ostéochondrose}

Même si l'ostéochondrose est un trouble généralisé à l'ensemble du squelette, il est évident que des conditions locales aggravantes sont responsables des anomalies cartilagineuses, des ostéonécroses et donc des symptômes observés. Parmi ces conditions, il faut sans doute retenir en premier lieu l'agression purement mécanique résultant soit d'une fragilité de l'os ou de l'articulation (d'origine nutritionnelle ou génétique), soit d'une trop forte surcharge localisée (résultant d'un excès de poids, d'une mauraise conformation ou d'une mauvaise position debout):

\section{Facteurs morphologiques ef statiques.}

a) Prédisposition spécifique. Comme le souligne Grøndalen, même chez les porcs non améliorés à faible vitesse de croissance le squelette est très souvent atteint d'ostéochondrose. Certaines zones sont plus facilement atteintes, ce qui résulte probablement du développement différentiel de l'organisme. Deux vagues de croissance se rencontrent au niveau des vertèbres lombaires (Hammond, 1961), rendant cette région particulièrement sensible. La soudure complète des épiphyses, selon Barone (1966), a lieu vers 6 ou 7 ans, alors qu'un porc atteint normalement $100 \mathrm{~kg}$ et est apte à la reproduction vers 7 ou 8 mois. L'immaturité du squelette est donc évidente chez les porcs engraissés et est particulièrement marquée en certains points : ainsi, la soudure des épiphyses n'est complète qu'à l'âge de 36 à 42 mois pour le fémur et est très tardive ( 6 à 7 ans) pour la tubérosité ischiale et la crête iliaque. Cela peut suffire à expliquer la fréquence de l'épiphysiolyse de la têłe du fémur et de l'apophysiolyse (rupture de la tubérosité ischiale). La fragilité du cartilage reliant l'ischium à l'apophyse a été signalée par Sabec et al. (1961) et Saunders (1972).

b) Facteurs génétiques.

- Conformation. Tous les auteurs ayant étudié ce syndrome s'accordent pour affirmer que la sélection des races à viande accentue l'immaturité relative du squelette au poids de 90 à $100 \mathrm{~kg}$. De plus, la conformation de l'animal est modifiée. Ainsi, à $35 \mathrm{~kg}$ de poids vif, le porc à viande sélectionné est plus long ( 3 à 5 vertèbres de plus et vertèbres plus grandes) de $20 \mathrm{~cm}$ environ, son centre de gravité est déplacé 
vers l'arrière étant donné le poids du jambon, l'ischium est plus court et la cavité cotyloïde logeant la tête du fémur est moins profonde (Koch et Meister, 1972). Toutes ces caractéristiques du porc moderne conduisent à une posture voûtée, défavorable aux articulations des membres (surtout postérieurs) et des vertèbres lombaires.

Selon Koch ef Meister (1972), l'épaississement du col du fémur dû à une plus forte pression et l'augmentation de 3 à $5^{\circ}$ de l'angle de torsion du col et de l'épiphyse, seraient favorables à l'épiphysiolyse en créant de nouvelles trajectoires d'ossification ef des forces cisaillantes. La proportion relative d'os par rapport au muscle serait également plus faible chez le Porc Piétrain (Dumont et al., 1969), chez lequel la fréquence des troubles locomoteurs est grande. Enfin, selon Günther et Rosin (1971), la minéralisation osseuse des membres postérieurs est relativement faible par rapport à celle du reste du squelette chez le Porc Landrace actuel, cette observation étant confirmée par Berschneider (1972).

La forme de l'articulation, influant sur sa stabilité, a été particulièrement étudiée par Grøndalen qui lui accorde une importance prépondérante et propose de l'employer comme critère de sélection. L'articulation du grasset en particulier aurait une forme favorisant une surcharge locale sur le condyle médian du fémur par l'intermédiaire de la partie médiane de l'éminence intercondyloïde du tibia.

En résumé, les caractéristiques constitutionnelles du Porc en font une espèce plus sensible que d'autres vis-à-vis des traumatismes osseux et articulaires et cette faible vigueur générale est encore aggravée par la pression de sélection.

Chez les races porcines très sensibles au SFP, l'héritabilité moyenne de ce caractère serait de 0,2 à 0,4 (Pfleiderer, $1973 ;$ Teuscher, 1972).

- Vitesse de croissance. L'amélioration de la vitesse de croissance par la conjonction des facteurs génétiques et nutritionnels est très certainement la principale cause de l'augmentation de fréquence du syndrome de faiblesse des pattes. Elle conduit en effet, non seulement à un fort accroissement de la charge que l'appareil ostéo-articulaire doit supporter, mais aussi à une plus grande immaturité du squelette à poids corporel égal. Selon Grøndalen (1976), si l'incidence de l'ostéochondrose est encore très élevée (95 p. 100) chez les porcs à croissance lente, le degré de sévérité des lésions est beaucoup plus grand chez les porcs à croissance rapide. Dans les observations effectuées par cet auteur il existe d'ailleurs une très forte corrélation entre la vitesse de croissance à $60 \mathrm{~kg}$ ef les troubles locomoteurs; à $100 \mathrm{~kg}$, Walker ef al. (1966) ont produit une dégénérescence fibreuse des cartilages de l'épiphyse distale du cubitus par de simples surcharges pondérales sur les animaux.

Fritschen (1973) note une forte corrélation négative entre le gain journalier et la résistance à la rupture des os longs.

\section{Facteurs nutritionnels.}

a) Les apports énergétiques ef azotés qui déterminent pour une grande part la vitesse de croissance des animaux influent évidemment sur la fréquence de la faiblesse des pattes. Cependant, leur influence sur l'incidence de l'ostéochondrose serait moins claire (Grondalen, 1976).

b) L'alimentation minérale a longtemps faił figure de principale accusée dans l'étiologie de ces troubles locomoteurs. En fait, des apports élevés ou faibles de cal- 
cium et de phosphore, associés à un apport normal de vitamine $D$, ne semblent pas modifier la fréquence des troubles observés (Grøndalen, 1976 ; Duthie et Lancaster, 1964 ; Walker et al., 1966). Des apports phosphocalciques très élevés augmenteraient même l'incidence de la faiblesse des pattes (Berschneider, 1972).

Plusieurs auteurs ont étudié les besoins phosphocalciques comparés des porcs à viande améliorés et des porcs de fype ancien. Nous avons déjà cité des travaux signalant le retard relatif de minéralisation des os des membres postérieurs (Günther et Rosin, 1971) ou des extrémités (Berschneider, 1972) chez le porc moderne, dont les besoins seraient supérieurs à ceux du porc ancien (Cuthbertson et Pomeroy, 1962 ; Meyer et Groning, 1965 ; Kirchgessner et Burgstaller, 1966 ; Günther et Rosin, 1971). Cependant, les apports couramment pratiqués en alimentation porcine permettent toujours de couvrir largement les besoins ainsi définis, avec un bon accord entre auteurs (A.R.C., 1967 ; N.R.C., 1973 ; Coalson et al., 1972 ; Cromwell et al., 1972 ; Bayley et al., 1975 ; Reinhard et al., 1976).

Les recommandations moyennes, par $\mathrm{kg}$ de ration sèche, issues de ces travaux vont de $9,5 \mathrm{~g}$ de $\mathrm{Ca}$ et $7,5 \mathrm{~g}$ de $\mathrm{P}$ en début de croissance à $7 \mathrm{~g}$ de $\mathrm{Ca}$ et $6 \mathrm{~g}$ de $\mathrm{P}$ en fin d'engraissement.

D'autres nombreuses observations portant sur les os atteints d'ostéochondrose ne révèlent pas de diminution du degré de minéralisation (teneurs en cendres, $\mathrm{Ca}$ ef P) par rapport à des os normaux (Englert ef al., 1967 ; Stadie, 1971 ; Weiss ef al., 1973 ; Röpke, 1973 ; Afschar-Hamdi, 1973). Cependant, ces deux derniers auteurs signalent une teneur plus faible en magnésium dans les os atteints de lésions.

Enfin, des apports phosphocalciques beaucoup plus faibles que les apports couramment recommandés ne semblent pas augmenter les cas de boiterie, bien que le degré de minéralisation ef la résistance à la rupture des os soient considérablement diminués (Stockland et Blaylock, 1973). Des troubles osseux généralisés, du type ostéodystrophie fibreuse, provoqués par des apports faibles de calcium et de phosphore, ne sont pas toujours associés à des boiteries sévères (Doige ef al., 1975) ni à des lésions de dégénérescence articulaire.

L'excès relatif de chlore (sous la forme de chlorure d'ammonium) par rapport au potassium et au sodium, et l'acidose métabolique qui en résulte, augmentent chez les volailles la fréquence de la dyschondroplasie tibiale caractérisée par une prolifération anormale des cartilages de la tête du tibia (Leach et Nesheim, 1972 ; Sauveur et Mongin, 1974). Bien que le type d'anomalie ne soit pas toujours comparable dans l'ostéochondrose du Porc, il est tentant d'attribuer un rôle au déséquilibre acidobasique. Des essais en lots (en collaboration avec l'I.T.P. ef I'E.D.E. du Finistère) n'ont pas montré d'influence défavorable de l'incorporation de 0,5 p. 100 de chlorure d'ammonium dans le régime.

De même, rien ne permet de penser qu'il s'agirait d'une ostéodystrophie rénale due à une hydroxylation insuffisante de la vitamine $D_{3}$.

Enfin, aucun travail ne permet pour le moment de mettre en cause une insuffisance en certains oligo-éléments $(\mathrm{Mn}, \mathrm{Zn}, \mathrm{Cu}, \ldots)$ dans l'apparition de la faiblesse des pattes chez le Porc. Seul le sélénium pourrait intervenir indirectement en agissant sur la qualité du muscle. 


\section{Facteurs écologiques.}

La densité de peuplement, qui détermine la possibilité d'exercice, a été citée par plusieurs auteurs comme étant un facteur primordial dans le SFP (Lefebvre ef al., 1975). Il est bien connu que les déplacements favorisent la nutrition du cartilage articulaire par diffusion et engendrent les forces directrices de l'ossification. Cependant, Grøndalen (1976) ne remarque pas de diminution du degré d'ostéochondrose par l'exercice et attribue son effet bénéfique aux systèmes musculaire et nerveux.

La nature du sol ou du parquet est également importante, car elle détermine la forme et la qualité des onglons et influe sur la posture des animaux. Un sol dur et rugueux ou un plancher en caillebotis mal espacés irrite les onglons. Un sol lisse favorise les glissades qui traumatisent les articulations. Cette influence du sol sur les onglons, pouvant aussi aboutir à des arthrites infectieuses, a été bien étudiée par Fritschen (1973) mais n'entre pas dans le cadre de cette revue. Il n'est d'ailleurs pas démontré que la fréquence ou le degré de gravité des lésions d'ostéochondrose en soit concerné.

\section{Aspects anatomopathologiques de l'ostéochondrose}

\section{Aspects cliniques.}

a) Stade d'apparition. Ce syndrome apparaît à tout âge (Grøndalen, 1974b), la plus grande fréquence se situant entre 60 ef $90 \mathrm{~kg}$ sur le Landrace Allemand (Grünhagen et al., 1970 ; Teuscher, 1972). D'autres auteurs (Stadie, 1971 ; Vaughan, 1971 ; Neuffer, 1972 ; Ghaffari-Kaschani, 1973) estiment que les anomalies de position et de mouvement interviennent entre 30 et $50 \mathrm{~kg}$ et qu'elles s'accentuent au fur et à mesure que le poids vif augmente. En Norvège (Grondalen, 1976), 35 p. 100 des verrats de sélection sont réformés avant 1,5 ans pour « faiblesse des pattes ».

b) Localisation. Les anomalies sont plus fréquentes sur le train arrière (Pfleiderer, 1973), que sur le train avant. Les articulations des pattes avant touchées sont celles du carpe, du métacarpe et des phalanges. Les modifications articulaires entraînent des positions anormales et diverses des pattes avant (arqûre, boiteries, animal cagneux ou panard, flexion des articulations, etc...). Il en est de même pour les pattes arrières, les manifestations étant plus marquées : raideur des pattes au début, jusqu'à impossibilité de la station debout, mouvement de balancement lors des déplacements, parfois position du «chien assis » (paraplégie complète ou partielle, unilatérale ou bilatérale, notamment chez la Truie).

Les anomalies des onglons peuvent aussi entraîner des modifications de posture, mais elles ne sont pas reliées à de l'ostéochondrose comme pour le SFP où les déformations articulaires sont essentielles.

\section{Aspects macroscopiques.}

a) Types de lésions.

Deux groupes importants, ostéochondrose et arthrose, sont décrits par Goedegebuure (1976), en fonction de leur localisation : dans l'ostéochondrose, l'os est 
atteint à proximité des cartilages, soit articulaires (atrophie de la tête du fémur par exemple), soit de conjugaison (cas des épiphysiolyses dont celle de la tête du fémur). L'os atteint est donc de l'os épiphysaire ou métaphysaire, l'os diaphysaire étant rarement touché. Il y a modification de l'ossification endochondrale ef de l'os sous-chondral sous-jacent aux cartilages articulaires. Dans l'arthrose, les couches superficielles et/ou profondes des cartilages articulaires ou du noyau pulpeux des disques intervertébraux sont atteintes. Macroscopiquement, la différence porte sur l'aspect visible des lésions articulaires tandis qu'une coupe sagittale est nécessaire pour constater les lésions osseuses ef ostéochondrales profondes. Les deux formes de lésions coexistent en général dans la faiblesse des pattes.

b) Répartition anotomique des lésions.

- Les plateaux épiphysaires les plus fréquemment atteints sont ceux du radius distal et du cubitus distal, du fémur proximal et de l'humérus proximal (épiphysiolyse) et de la tubérosité ischiale (apophysiolyse).

- Les articulations les plus touchées sont celles du grasset (condyle médian du fémur distal), du coude (condyles latéral et médian de l'humérus distal, et semilunaire), de l'épaule (tête de l'humérus et acetabulum de l'omoplate), de la hanche (tête du fémur), du carpe (radius distal), du jarret (surface articulaire du tibia distal, os tarsiens), de la colonne vertébrale (vertèbres lombaires). Selon Goedegebuure (1976) et Grøndalen (1976), l'articulation la plus fréquemment lésée est celle du grasset (ou genou).

c) Signes radiologiques. Ils sont variés puisque l'on peut observer des décalcifications (os métaphysaire) ou des calcifications anormales (ostéosclérose ef ostéophytose, ossification du cartilage de conjugaison). On peut également observer des déformations osseuses : atrophie de la tête fémorale ou humérale, changement d'orientation des condyles, disparition des crêtes tibiales, changement de l'angle articulaire ; toutefois, les examens radiologiques sont souvent effectués post-mortem et ne servent généralement pas dans le diagnostic.

d) Examen des os et des articulations. A l'œil nu, on peut voir les dysplasies de la tête du fémur et de l'humérus, l'atrophie des cartilages articulaires, leur absence ou la diminution de leur épaisseur, l'aplatissement de la fête articulaire (condyles fémoraux), les fissures du cartilage et les invaginations (radius ef tibia), des lésions hémorragiques superficielles du cartilage de conjugaison, les dysjonctions chondro-osseuses avec perte de substance osseuse, «l'arthrosis deformans» (apophysiolyse des condyles du tibia distal), l'épiphysiolyse (décollement) de la tête du fémur, la rupture de la tubérosité ischiale et l'apparition de tissu fibreux à la place du cartilage de conjugaison.

\section{Examen histologique.}

a) Au niveau tissulaire. Les principales lésions rencontrées sont l'ossification du cartilage de conjugaison, les fissures macro-et microscopiques dans le cartilage articulaire, des processus de nécrose ef de fibrose, des kystes, la perte de substance trabéculaire, une prolifération anormale de chondrocytes, de l'ostéoclasie. 
b) Au niveau cellulaire. Dans la substance fondamentale, il y a apparition de structures vacuolaire et granuleuse ef une modification des fibrilles.

Au niveau des chondrocytes, il peut y avoir dégénérescence ef nécrose, prolifération anormale et défaut de maturation conduisant à l'atrophie et à l'hypoplasie du cartilage articulaire avec une ossification sous-chondrale insuffisante, ce qui provoque l'aplatissement du cartilage articulaire.

On peut observer (Goedegebuure, 1976) des figures de régénérescence (formation de «broad-capsules » et d'un chondroblastème, résorption des lésions complète ou partielle) ou de dégénérescence par remplacement du tissu fibreux ef disparition plus ou moins complète du cartilage et de l'os sous-jacent (Meijer et al., 1975).

Dans l'os sous-chondral, il apparaît des microfractures de l'os trabéculaire ef du cartilage calcifié, de la myélofibrose, de l'ostéoclasie, un collapsus de la couche externe du cartilage, un accroissement de la formation d'os nouveau et des changements vasculaires. Toutes ces modifications peuvent aller jusqu'à la nécrose aseptique de la moelle et de l'os.

c) Vascularisation. L'examen des artères de la tête fémorale montre chez les porcs atteints d'épiphysiolyse (fémur) une fibrose artérielle et une hypertrophie de la média. La lumière artérielle est diminuée ou obstruée et, en conséquence, l'ostéogenèse est perturbée (Nemeth ef Van der Valk, 1976).

4. Précisions sur les principales lésions. (Une revue des lésions principales a été donnée par Lefebvre et al., en 1975.)

a) Cartilages articulaires. Selon Thurley (1965), il existe trois types essentiels de lésions :

- lésion en gouttière avec cartilage mince et lisse, surtout pour le fémur et l'humérus ; - lésion proliférative avec zone cartilagineuse superficielle très irrégulière (épiphyse distale du fémur) ;

- lésion surélevée avec décollement du cartilage, altération du cartilage calcifié en voie d'ossification (cubiłus).

b) Lésion du cubitus (ulna). Il s'agit surtout de lésions épiphysaires des faces distale et proximale du cubitus. On distingue des stades ou types principaux :

- lésion au niveau du cartilage de conjugaison entre les zones de croissance et d'ossification ;

- lésion dans la zone provisoire de calcification avec fracture, déplacement du cartilage calcifié, hémorragies et dégénérescence fibreuse ;

- processus de réparation avec réduction des lésions à une bande fibreuse ou à un nodule avec parfois résorption totale.

Il n'existe pas de corrélation entre l'apparition du syndrome el la gravité des lésions (Walker et al., 1966 ; Vaughan, 1971 ; Thurley, 1967 et 1969 ; Fell ef al., 1970).

c) L'épiphysiolyse de la tête du fémur. Le décollement épiphysaire de l'articulation de la hanche est une des manifestations caractéristiques du SFP (on rencontre aussi cette lésion chez l'Homme ef le Chjen). L'examen histologique révèle une nécrose du cartilage de croissance et de l'os sous-jacent. Cette anomalie n'est pas très fréquente. 
d) L'apophysiolyse de la fubérosité ischiale. Cette apophysiolyse peut être unilatérale ou bilatérale et a été bien décrite par Saunders (1972), surtout chez les truies de $2^{\mathrm{e}}$ et $3^{\mathrm{e}}$ portée ef en gestation. En cas de rupture bilatérale, la truie est en position du « chien assis ». Selon Saunders, l'os reliant l'apophyse à l'os principal a été soit résorbé et remplacé par du cartilage, soit jamais complètement formé durant la croissance.

\section{Conclusion générale}

Des lésions d'ostéochondrose aseptique sont présentes de façon quasi générale chez les porcs sélectionnés de $100 \mathrm{~kg}$ mais sont également très fréquentes chez les porcs plus jeunes. Les zones particulièrement touchées sont les condyles médians du fémur et de l'humérus distaux, le plateau épiphysaire distal du cubitus et les articulations intervertébrales lombaires. La présence de ces lésions ne se traduit pas toujours par l'apparition du syndrome de faiblesse des pattes, mais leur aggravation peut conduire à l'arthrose, à la dégénérescence des disques vertébraux et à l'épiphysiolyse.

La responsabilité des facteurs purement mécaniques dans l'ostéochondrose et ses conséquences semble bien démontrée et la cause principale serait la surcharge locale traumatisante. Les conditions aggravantes sont la mauvaise conformation corporelle, l'instabilité de certaines articulations, la forte vitesse de croissance, l'habitat et en particulier le sol. Il faut y ajouter le manque d'exercice, bien qu'il ne semble pas influer directement sur le degré de sévérité des lésions osseuses.

Les facteurs alimentaires et notamment le niveau des apports phospho-calciques, doivent être mis hors de cause, tandis que les facteurs génétiques joueraient un rôle non négligeable.

Réunion Groupe Développement INRA/Productions animales Montpellier, 17-18 mai 1977.

\section{Références}

AFSCHAR-HAMDI K., 1973. Colcium, Phosphor-und Mognesiumgehalt ousgewählter Knochenproben beim Schwein unter Berücksichtigung verschiedener Gewichtsklassen, Rassen und des Beinschwäche - Syndroms. Diss. Dokt. Agrarwiss., Hohenheim.

Agricultural Research Council, 1967. The nutrient requirements of farm livestock, n 3. Pigs. H.M.S.O., London.

BARONE R., 1966. Anatomie comporée des mammifères domestiques. Ostéologie, 1, Ec. Nat. Vét. Lyon, Lab. anat.

BAYLEY H. S., ARTHUR D., BOWMAN G. H., POS J., THOMPSON R. G., 1975 . Influence of dietary phosphorus level on growth and bone development in boars and gilts. J. anim. Sci., 40, 864-870.

BERSCHNEIDER F., 1972. Biochemische Untersuchungen zum Problem der Bewegungsanomalien des Fleischschweines, 89-158. In Gesundheilliche Aspekfe der Fleischschweinproduktion. Stellungs und Gliedmasseanomalien. V.E.B. Gustav Fischer Veriag, Jena.

COALSON J. A., MAWXELL C. V., HILLIER J. C., WASHAM R. D., NELSON E. C., 1972. Calcium and phosphorus requirements for young pigs reared under controlled environmental conditions. J. Anim. Sci., 35, 1194-1200.

CROMWELL G. L., HAYS V. W., SCHERER C. W., OVERFIELD J. R., 1972. Effects of dietary calcium and phosphorus on performance and carcass, metacarpal and turbinate characteristics of swine. J. Anim. Sci., 34, 746-751. 
CUTHBERTSON A., POMEROY R. W., 1962. Quantitative anatomical studies of the composition of the pig at 50,68 and $92 \mathrm{~kg}$ carcass weight. II. Gross composition and skeletal composition. J. agric. Sci. Camb., 59, 215-223.

DOIGE C. E., OWEN B. D., MILLS J. H. L., 1975. Influence of calcium and phosphorus on growth and skelefal development of growing swine. Can. J. Anim. Sci., 55, 147-164.

DUMONT B. L., SCHMITT O., ROY G., 1969. Développement musculaire comparé de porcs Piétrain et Large White. Rec. Méd. vet., 145, 937-947.

DUTHIE I. F., LANCASTER M. C., 1964. Polyarthritis and epiphysiolysis of pigs in England. Vet. Rec., 76, 263-272.

FELL B. F., LEIGH L. C., WENHAM G., ELSLEY F. W. H., 1970. Observations on the incidence of spontaneous lesions of bone in pigs. Br. vet. J., 126, 306-315.

FRITSCHEN R., 1973. A product of confinement. Hog Farm Management. June 1973.

GHAFFARI-KASCHANI H., 1973. Histologische Untersuchungen am Gelenkknorpel des Schweines unter Berücksichtigung verschiedener Gewichtsklasse, Rassen und des Beinschwäche-Syndroms. Diss. Dokt. Agrarwiss., Hohenheim.

ENGLERT H. K., KOPP C., GROSSMANN W., 1967. Orientierende Untersuchungen über Knochenaschegehalte von Schweinen mit und ohne Bewegungsstörungen. Tierärztl. Umsch., 22, 219-220.

GOEDEGEBUURE S. A., 1976. Macroscopical and microscopical features in porcine osteochondropathies. Proc. 3rd int. Conf. Prod. disease in farm animals. Wageningen, sept. 13-16.

GRONDALEN T., 1974a. Osteochondrosis, arthrosis and leg weakness in pigs. Nord. Vet. Med., 26, 534-537.

GRONDALEN T., 1974b. Osteochondrosis, arthrosis in pigs. I. Incidence in animals up to $120 \mathrm{~kg}$ live weight. Acta vet. scand., 15, 1-25.

GRONDALEN T., 1974c. Osteochondrosis, arthrosis in pigs. II. Incidence in breeding animals. Acta vet scand., 15, 26-42.

GRONDALEN T., 1974d. Osteochondrosis, arthrosis in pigs. III. A comparison of the incidence in young animals of the norwegian Landrace and Yorkshire breeds. Acfa vet, scand., 15, 43-52.

GRONDALEN T., 1974e. Osteochondrosis, arthrosis in pigs. IV. Effect of overloading on the distal epiphyseal plate of the ulna. Acta ret. scand., 15, 53-60.

GRONDALEN T., 1974f. Osteochondrosis, arthrosis in pigs. VI. Relationship to feed level and calcium, phosphorus and protein levels in the ration. Acto vef. scond., 15, 147-169.

GRONDALEN T., 1974g. Osteochondrosis, arthrosis in pigs. VII. Relationship to joint shape and exterior conformation. Acta Vet. Scand., Supp. 46, 1-32.

GRONDALEN T., 1974h. Leg weakness in pigs. I. Incidence and relationship to skeletal lesions, feed level, protein and animal supply, exercise and exterior conformation. Acta vet. scand., 15, 555-573.

GRONDALEN T., 1976. Viewpoints on the porcine leg weakness syndrome. Proc. 3rd int. Conf. Prod. disease in farm animals. Wageningen, sept., 13-16.

GRONDALEN T., VANGEN O., 1974. Osteochondrosis, arthrosis in pigs. V. A comparison of the incidence in three different lines of the norwegian Landrace breed. Acto vet. scand., 15, 61-79.

GRÜNHAGEN H., STEINHAUF D., WENIGER J. M., 1970. Untersuchungen zum BeinschwächeSyndrom beim Mastschwein. Züchtungskunde, 42, 374-383.

GÜNTHER K., ROSIN G., 1971. Uber die Ansatz-Kapazitat des Fleischschweines für Mineralstoffe in Verlauf des Wachstums. 2. Wachstum und Entwicklung der einzelnen Skeletteile. Z. Tierphysiol. Tierernähr. Fuftelmittelk., 27, 134-150.

HAMMOND J., 1961. La reproduction, la croissance ef l'hérédité des animaux à la ferme. Le Porc. Vigot Frères, Paris.

HERRMANN H. J., 1972. Zur Pathomorphologie, Pathogenese und Atiologie der Osteoarthropathien des Schweines. Arch. exp. Vet.-Med., 26, 618-644.

KIRCHGESSNER M., BURGSTALLER G., 1966. Der Wachstumsquotient von Mastschweinen neuer Zuchtrichung. Züchtungskunde, 38, 176-179.

KOCH T., MEISTER R., 1972. Anatomische Untersuchungen am Fleischschwein unter besonderer Berücksichtigung des passiven Bewegung sapparates, 23-48. In Gesundheitliche Aspekte der Fleischschweinproduktion. Stellungs und Gliedmasseanomalien. V.E.B. Gustav Fischer Verlag, Jena. 
LEACH R. M. Jr., NESHEIM M. C., 1972. Further studies on tibial dyschondroplasia (cartilage abnormality) in young chicks. J. Nutr., 102, 1673-1680.

LEFEBVRE A., RUNAVOT J. P., KERISIT R., 1975. Le syndrome de la faiblesse des pattes chez le Porc. Inst. Tech. Porc., Paris, Pp. 71.

MEIJER P., GOUDS-WAARD J., GOEDEGEBUURE S. A., BUDHAI S., 1975. Immunological, bacteriological and morbid-anatomical feature of arthrosis/arthritis of the stifle joint in swine. T. Diergeneesk., 100, 1109-1116.

MEYER H., GRÖNING M., 1965. Untersuchungen am Autopodiumskelett des Schweines. 2. Mitt. Asche- und Mineralstoffbestimmungen. Züchlungskunde, 37, 224-232.

NEMETH F., VAN DER VALK P. C., 1976. Vascular lesions in epiphysiolysis capitis femoris in swine. Proc. 3rd. intern. Conf. Prod. disease in farm animals. Wageningen sept. 13-16.

NEUFFER K., 1972. Bedeutung der Bewegungsanomalien beim Fleischschwein und Darstellung des Untersuchungsmaterials. 13-22. In gesundheitliche Aspekfe der Fleischschweinproduktion. Stellungs und Gliedmosseanomalien. V.E.B. Gustav Fischer Verlag, Jena.

National Research Council, Nat. Acad. Sci., 1973. Nuirient requirements of domestic animals. 2. Nutrient requirements of swine, 7th ed., Washington D.C.

PFLEIDERER U. E., 1973. Zum Einfluss der Hinterhandschwäche auf die Mastleistung bei Mastprüfungsschweinen. Schweinezucht u. Schweinemast, 10, 286-288.

REINHARD M. K., MAHAN D. C., WORKMAN B. L., CLINE J. H., FETTER A. W., GRIFO A. P. Jr., 1976. Effect of increasing diefary protein level, calcium and phosphorus on feedlot performance, bone mineralization and serum mineral values with growing swine. J. Anim. Sci., 43, 770-780.

RÖPKE H., 1973. Uber den Kalzium-Phosphor- und Magnesium-Gehalt gesunder und arthrotisch veränderter Tarsalknochen des Schweines. Diss. Dokt. Med. Veter. Hannover.

SABEC Von D., SCHILLING E., SCHULZ L. C., 1961. Eine Arthrosis deformans des Sprunggelenkes beim Schwein. Dtsch. tierarztl. Wschr., 68, 231-236.

SAUNDERS C., 1972. When sows can't stand the strain. Pig Farming, may 1972., 43-44.

SAUVEUR B., MONGIN B., 1974. Influence of dietary levels of chloride, sodium and potassium on chick cartilage abnormalities. Proc. 15th WId. Poultry Congr., New Orléans, 180-181.

STADIE U., 1971. Gewicht, Volumen und Aschegehalt gesunder und arthrotish veränderfer Tarsalknochen des Schweines. Diss. Dokt. Med., Vet., Hannover.

STOCKLAND W. L., BLAYLOCK L. G., 1973. Influence of dietary calcium and phosphorus levels on the performance and bone characteristics of growing-fivishing swine. J. Anim. Sci., 37, 906-912.

TEUSCHER T., 1972. Untersuchungen über das Beinschwächesyndrom on einer Zuchtpopulation des Hausschweines (Deutsche Landrasse). Diss. Dockt. Landbauwiss., Berlin.

THURLEY D. C., 1965. Arthropathy in pigs. Proc. roy. Soc. Med., 58, 369-370.

THURLEY D. C., 1967. Muskulaturentwicklung und Gliedmassenschwäche (leg weakness) bei Schweinen. Disch. fierärztl. Wschr., 74, 336-338.

THURLEY D. C. 1969. Changes in the epiphysial cartilage of immature pigs without clinical symptoms. Path. vet., 6, 217-226.

VAUGHAN L. C., 1969. Locomotory disturbance in pigs. Br. vet. J., 125, 354-365.

VAUGHAN L. C., 1971. Leg weakness in pigs. Vet. Rec., 89, 81-85.

WALKER T., JONES A. S., 1962. A study in leg weakness in boars. Anim. Prod., 4, 297 (abstr.).

WALKER T., FELL B. F., JONES A. S., BOYNE R., ELLIOTT M., 1966. Observations in leg weakness in pigs. Vet. Rec., 79, 472-479.

WEISS G. M., DEKKER Th. P., VAN PUTTEN G., SYBESMA W., 1973. Association of blood and bone composition and postmortem muscle changes to leg weakness, in Dutch Landrace swine. J. Anim. Sci., 37, 974-978. 